\title{
Reducing sedentary behavior and cardiovascular disease risk factors in office workers: a study protocol
}

\author{
Zhixiong Zhou ${ }^{1, \# *}$, Shanshan Dong ${ }^{1}$, Hong Ren ${ }^{2}$ and Zenong Yin ${ }^{1,3, \#}$ \\ ${ }^{1}$ Institute for Sport Performance and Health Promotion, Capital University of Physical Education and Sports, Beijing, China \\ ${ }^{2}$ School of Sport Sciences, Beijing Sport University, Beijing, China \\ ${ }^{3}$ Department of Kinesiology, Health and Nutrition, The University of Texas at San Antonio, San Antonio, Texas, USA \\ \#These authors contributed equally to this work
}

\begin{abstract}
Background: Sedentary lifestyle are public health concerns that threatening human health, and sedentary behavior(SB) intervention in the workplace has attracted wide attention in the field of public health.

Methods: A quasi-experimental design with randomized treatment assignment will be used to test the effect of breaking up prolonged sitting during work hours on SB and cardiometabolic risk in professional office workers in China. There are two intervention protocols: A) Interrupting SB prompted by timer app every 60min of sitting during work hours; B) Participating in 30 min of moderate to vigorous physical activity at work. The intervention will be implemented in 2 worksite locations for 12 months. Study outcomes include SB and physical activity measured by accelerometry, and cardiometabolic risk factors and will be assessed at baseline and 12-month posttest.
\end{abstract}

Discussion and conclusion: The intervention protocols integrate a few health behavior promotion theories, will provide a reference for selecting appropriate intervention measures to conduct an effectiveness on SB interruption at work, decrease in SB duration, and influence on cardiovascular risk factors.

\section{Introduction}

Sedentary behavior (SB) is currently a global lifestyle phenomenon that have associated with reduction in energy expenditure independent of moderate-to-vigorous physical activity (MVPA) [1,2]. SB refers to human activities in sitting or reclining position with energy expenditure less than 1.5 Metabolic Equivalents (METs) during waking hours [3]. Prolong time spent in SB is significantly correlated with some indices threatening human health, such as cardiovascular risk factors [4], type II diabetes, obesity, cardiovascular diseases, breast cancer and colon cancer [5,6]. Total duration of SB is correlated with all-cause mortality [7].

In recent years, $\mathrm{SB}$ intervention in the workplace has attracted wide attention in the field of public health. Two recent systemic reviews $[8,9]$ reported that majority of the interventions have mainly focused on decreasing the prolonged sitting time by breaking sitting with periodic prompts [10-12], promoting changes in sitting position with sit-stand workstations [13-20], and combining work with low-intensity physical activity (e.g., treadmill working station).

There is inconsistent evidence that the interventions have reduced time spent in SB and produced positive effects on cardiometabolic risks and work productivity $[21,22,8]$. It was also pointed that the studies have been overwhelmingly conducted in the developed countries [8]. Finally, the 2016 Cochrane review found no RCT that examined the effects of interrupting prolonged sitting on SB, physical activity and cardiometabolic risks [8].

Prevalence of SB has been widely reported among urban residents and professional worker in China $[23,24]$ amid the report of low levels of the physical activity of office workers during work hours [25]. We recently reported that Chinese professional office workers spent over 9 hours in SB and those in the most sedentary category were four times more likely to develop metabolic syndrome, independent of participation in MVPA at one-year follow-up [26]. The present study aims to test the effects of two workplace intervention protocols on the duration of SB during working hours and reductions of cardiovascular risk factors in Chinese professional office workers.

\section{Methods}

Study Design and Sample: This is a three-arm quasi-experimental design with randomized treatment assignment conducted in three worksites in Beijing, China. Each of the three worksites will be randomly assigned to one of the intervention arms. Study outcomes will be assessed at baseline and 12-month posttest.

The worksites included in this study are one university, one government research institute, and a private scientific research and developed firms. All current employees aged 30-60 years are invited to participate in the study. Exclusion criteria are as follows: BMI $\geq 28$ and diagnosis of hypertension, coronary heart disease, diabetes, arthritis,

Correspondence to: Zhixiong Zhou and Zenong Yin, Institute for Sport Performance and Health Promotion, The Capital University of Physical Education and Sports, China, E-mail: zhou126@126.com, Zenong.yin@utsa.edu

Key words: sedentary time, workplace intervention, moderate to vigorous physical activity, physical function training

Received: January 13, 2018; Accepted: January 30, 2018; Published: February 02,2018 
or joint muscle pain. Those who have plans to travel for business or to be absent from work $>4$ weeks during the 12 -month study period will also be excluded. The participants should spend at least $60 \%$ of the time at work during working hours. All study participants need to sign the informed consent form.

We expected a moderate effect on the reduction of times spent in $\mathrm{SB}$ after the intervention based on published studies [11,27,28]. Using twosided test, we need to recruit 210 participants to detect significant change in SB with $80 \%$ power and significance level less than 0.05 [29]. The study was approved by the Ethics Committee of the Capital University of Physical Education and Sport University (ChiCTR-TRC-14005239).

\section{Description of Intervention}

Theoretical model: According to the Healthy Workplace Framework and Model proposed by WHO [30], the intervention targets individual-, environment- and organization-level factors to reduce SB in the professional office workers with multicomponent and multi-dimensional intervention. We will use the Health Action Process Approach [31] and behavior incentives [32] to improve social support and promote self-monitoring and self-management. Multi-component interventions have consistently demonstrated effect in reducing sedentary time in studies conducted in the developed countries [8].

Intervention Design: Group A: This is the intervention arm of the study. Office workers will stand up and do 10-min of light- or moderateintensity physical activity after every 60 -min of sitting, prompted by a visual signal and sound on desk computer screen which is generated by a computer software (Workrave, Free Software Foundation, Inc, http:// www.workrave.org/). This intervention is designed to interrupt SB by breaking up prolonged sitting every $60 \mathrm{~min}$ and prompt participants to be physically active for $10 \mathrm{~min}$. This intervention is expected to reduce the cardiovascular-metabolic risk by replacing SB with lower intensity physical activity [33,34].

Group B: This is the active control arm of the study. Daily group physical activity sessions as work breaks will be offered to the workers for 10-15 min in the morning and afternoon to guarantee 30-min moderate physical activity daily. This intervention is expected to reduce the cardiovascular-metabolic risk by introducing the 30 -min MVPA [35].

Control Condition: This is the non-intervention control arm of the study. The participants are informed that the study aims to longitudinally observe the correlation of physical activity and SB with cardiometabolic risk measures. There is no exercise intervention for the control group.

Intervention implementation: The process of intervention implementation is depicted in (Figure 1). The objectives of the intervention for Group A and Group B are: 1) to motivate participation by learning the importance of healthy lifestyle and expected reduction of cardiometabolic risk, self-monitoring of $\mathrm{SB}$, and increasing selfefficacy for developing a healthy lifestyle; 2) to develop a personalized action plan for each participant to seek social support and overcome barriers); and 3) to prevent relapse by reviewing personal action plan and providing regular feedback and social support.

Orientation meeting: The participants will attend an orientation meeting and meet with a coach assigned to the worksite. The topics of the meeting will mainly include: introduction of the intervention and implementation process; development of personalized action plan, and discussion about the possible barriers and problems.

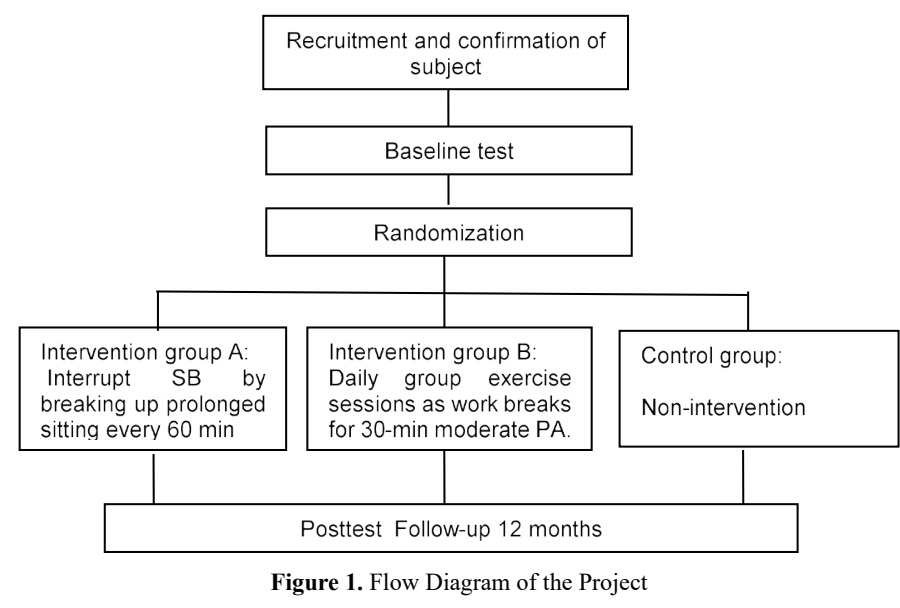

Expert lectures: Health experts will give 3 health education lectures on topics, such as importance of healthy lifestyle and consequences of SB on health outcomes, physical activity and exercise principles, and introduction of New Concept of Scientific Fitness for Office Workers which was created by our research team to provide a variety of physical activities designed for office setting.

Setting up social media support groups: Social media groups will be set-up for study participants at each worksite using a social media app (WeChat, Tencent Inc., Shenzhen city, China, https://web.wechat. com). One participant from each worksite will be selected as group leader to organize and monitor the group under the supervision of the worksite coach. The support groups have three functions: distributing tips on reducing SB and increasing physical activity; sharing experience of success and supporting each other among the group members; and sending out information and reminders of upcoming activities.

Implementation of exercise intervention: The timer App (Workrave) will be installed in the desk computer of each participant in Group A. After 60 min of use of the desk computer, the timer App will generate a series of pictures of stretching or callisthenic exercises with instructions in Chinese. The participants perform the exercises following the pictures on the screen for $10 \mathrm{~min}$. They can also use simple physical activities in New Concept of Scientific Fitness for Office Workers written by our research team. Instead of doing physical activities, the participants can also stand up to run an office errant or getting tea or water. The participants are instructed to gradually increase the amount and intensity of physical activity over time, without interfering their work productivity. After completing the break, the participants can close the timer reminder and resume work. We ask the participants to email a copy of exercise logs from the timer App to the research team every month. The participants in Group B will participate in 10-15 min session of MVPA as a work break led by the worksite coach at predetermined time in the morning and afternoon. The participants will do exercise routines with resistance band or dumbbells or calisthenics exercises inside the office or in the hallway.

Small group meetings: The participants will meet monthly (for 12 months) with the worksite coach to review personalized action plan and identify solutions for overcoming barriers.

Incentives and retention: To incentivize long-term participation and retain participants in the study, participants will receive a digital weight scale, digital pedometer, a fitness band set, and a sport jacket if they attend small group meeting (minimum of 8 meetings), email their timer App logs (minimum of 40 logs) in Group A, participants attend 
the daily group physical activity sessions with the frequency over $80 \%$ of the sessions in group B, and attend the posttest.

Study Measurements: The primary outcomes of the study are time (min/day) spent in SB, light physical activity and MVPA, and cardiometabolic risk measures. SB and physical activity will be objectively measured by Accelerometry (Actigraph GT3X-Plus, Pensacola, FL, USA), to measure time spent for light, moderate and vigorous activities, and SB during each day as well as work hours [11]. The participants will wear the accelerometers on the right hip from the time of waking up to bedtime for seven consecutive days. The data collected from the accelerometers will be analyzed by ActiLife 6 (Version 6.11.5, ActiGraph). Non-wear time will be set as 0 count at least within continuous $60 \mathrm{~min}$, and the counts within $0-100$ are allowed within $2 \mathrm{~min}$ [34]. To be considered as a valid measure, the accelerometer must be worn at least 10 hours/day for $\geq 4$ days, including one weekend day. SB is defined as average activity count $<100$ counts/minute. Light physical activity is defined as activity of 100-2296 counts/minute, and MVPA is $>2296$ counts/minute [36].

Cardiometabolic risk measures include fasting blood glucose (FBG), total cholesterol (TC), triglycerides (TG), high-density lipoprotein cholesterol (HDL-C), low-density lipoprotein cholesterol (LDL-C), anthropometric measures, and blood pressure (BP). Fasting venous blood will be used to analyze the cardiovascular risks. Automatic biochemistry analyzer (SynchronLX, Beckman Coulter, Brea, CA, USA) will be used to analyze blood glucose (oxidase method), HDL-C and TG (enzyme method). Height, weight and waistline will be measured in light clothing by trained research assistant. Diastolic and systolic blood pressure are measured with an electronic blood pressure monitor after 5-min rest.

Finally, demographic information (age, sex, income, etc.) and health history (e.g., diet, smoking, smoking, etc.) will be collected from the participants.

Data analysis: Descriptive statistics will be used to characterize the participants at baseline. The baseline data between the groups will be compared by analysis of variance. General Linear Model will be used to test the difference on SB, light physical activity, MVPA, and cardiometabolic risk measures between Group A and control group and between Group B and control group, controlling for covariates and baseline value of the outcome measure. Data will be analyzed using SPSS (version 21.0) with significance level at $\mathrm{p}<0.05$ (two-tailed)

\section{Discussion}

This study will compare the effects of two different intervention protocols on the SB and cardiovascular risk factors of office workers in the workplace, which will provide a reference for selecting appropriate intervention measures in the future. The multi-component approach used in this intervention more likely to engage the participants and produce the expected outcomes $[37,38]$.

Evidence supporting efficacy of SB reduction intervention by interrupting prolonged sitting with activity breaks is spars and suffers from methodological problems [8]. Previous studies demonstrated feasibility of reducing SB with reminders for active breaks and prompts for changing body positions $[12,39,40,41]$. However, the interventions were mostly small pilot studies with short follow-up [41] and none has examined impact on cardiometabolic risks $[13,19]$.

In the study, we will use incentives to improve the motivation of participating in the intervention activities and participant retention [42].
Such methods have been shown to be effective to increase participation in vaccination, cancer screening, tuberculosis examination, and quitting smoking [43] as well as lifestyle changes [44].

One significant strength of the study is the use of active control group (i.e., Group B) that controls non-specific intervention effect and maximize the interval validity of the study [45]. Longitudinal studies have demonstrated that $\mathrm{SB}$ is associated with increased risk for chronic disease and mortality independent of levels of MVPA. This assertion has not been tested in an experimental study. Examining the effect of Group B in the study will allow us to assess if reduction of SB is equally effective in lowering cardiometabolic risk without adding 30-min MVPA. Other strengths of the study include use of objective measurement of SB and physical activity [11-13,46-48], longer follow-up (i.e., 12-moth), and assessment of cardiometabolic risks as outcomes $[28,49]$.

\section{Conclusion}

Finding from this study can provide important information for designing worksite health promotion programs to reduce harmful effects of SB on cardiometabolic risk in professional office workers. The study also provides a test of multi-component SB reduction interventions in office workers in developing countries such as China.

\section{Competing Interests}

The authors declare that they have no competing interests.

\section{Funding}

This work was supported by 2015 Nationwide Physical Fitness Funding Project of General Administration of Sport of China (No. 2015B080). Trial registration: ChiCTR-TRC-14005239

\section{References}

1. Healy GN (2008) Television time and continuous metabolic risk in physically active adults. Med Sci Sports Exerc 40: 639-645. [Crossref]

2. Yang X (2008) The longitudinal effects of physical activity history on metabolic syndrome. Medicine and science in sports and exercise 40: 1424-1431. [Crossref]

3. Tremblay M (2012) Standardized use of the terms "sedentary" and "sedentary behaviours. Applied Physiology, Nutrition, and Metabolism 37: 540-542. [Crossref]

4. Shuval K (2014) Sedentary behavior, cardiorespiratory fitness, physical activity, and cardiometabolic risk in men: the cooper center longitudinal study. Mayo Clinic proceedings 89: . 1052-1062. [Crossref]

5. Thorp AA (2011) Sedentary behaviors and subsequent health outcomes in adults a systematic review of longitudinal studies. American journal of preventive medicine 41 : 207-215. [Crossref]

6. Tremblay MS (2010) Physiological and health implications of a sedentary lifestyle Appl Physiol Nutr Metab 35: 725-740.

7. Chau JY (2015) Sedentary behaviour and risk of mortality from all-causes and cardiometabolic diseases in adults: evidence from the HUNT3 population cohort. $\mathrm{Br} J$ Sports Med 49:737-742. [Crossref]

8. Shrestha N (2016) Workplace interventions for reducing sitting at work. Cochrane Database Syst Rev 3: CD010912.

9. Chu AH (2016) A systematic review and meta-analysis of workplace intervention strategies to reduce sedentary time in white-collar workers. Obes Rev 17: 467-481.

10. Cooley D, S. Pedersen (2013) A pilot study of increasing nonpurposeful movement breaks at work as a means of reducing prolonged sitting. Journal of environmental and public health 12: 128376 .

11. Healy GN (2013) Reducing sitting time in office workers: short-term efficacy of a multicomponent intervention. Preventive medicine 57: 43-48.

12. Evans RE (2012) Point-of-choice prompts to reduce sitting time at work: a randomized trial. American journal of preventive medicine 43: 293-297. 
13. Alkhajah TA (2012) Sit-stand workstations: a pilot intervention to reduce office sitting time. American journal of preventive medicine 43: 298-303. [Crossref]

14. Pronk NP (2012) Reducing occupational sitting time and improving worker health: the Take-a-Stand Project, 2011. Preventing chronic disease 9: E154.

15. Rodrigues VAJ (2012) Assessment of physical workload in boiler operations. Work (Reading, Mass.) 41: 406-413

16. Pigini L (2010) Designing reasonable accommodation of the workplace: a new methodology based on risk assessment. Disability and rehabilitation. Assistive technology 5: 184-198.

17. Ellegast R, Weber B, Mahlberg R (2012) Method inventory for assessment of physical activity at VDU workplaces. Work (Reading, Mass.) 41 Suppl 1: 2355-2359.

18. McLean L, Scott RN, Rickards J (2001) Computer terminal work and the benefit of microbreaks. Appl Ergon 32: 12.

19. Roelofs A (2012) The experience of musculoskeletal discomfort amongst bank tellers who just sit, just stand or sit and stand at work. Ergonomics 14: 10.

20. Hedge A (2004) Ithaca, Effects of an electric height-adjustable worksurface on selfassessed musculoskeletal discomfort and productivity in computer workers. Vol. Cornell University. NY: Cornell University.

21. Chau JY (2014) The effectiveness of sit-stand workstations for changing office workers' sitting time: results from the Stand@Work randomized controlled trial pilot. The international journal of behavioral nutrition and physical activity 11: 127 .

22. John D (2011) Treadmill workstations: a worksite physical activity intervention in overweight and obese office workers. Journal of physical activity \& health 8: 10341043.

23. Xu Jian, Zhou (2013) Survey of Physical Activity of Shenzhen Residents. Modern Preventive Medicine 3: 14.

24. Yang Gonghuan, Liu Na, Chen Aiping (2005) Survey of diet, physical activity and body mass index in Chinese population. Chinese Journal of Epidemiology 26: 6.

25. Xie Gaoqiang, Mai Liancheng, Beijing, Guangzhou (2008) The current situation of physical activity of urban and rural populations at work and their changes over the past 10 years. Journal of Health Research 37: 33.

26. Zhou Z (2016) Sedentary Behavior Predicts Changes in Cardiometabolic Risk in Professional Workers: A One-Year Prospective Study. J Occup Environ Med 58: e117-23.

27. Celis-Morales CA (2012) Objective vs. self-reported physical activity and sedentary time: effects of measurement method on relationships with risk biomarkers. PLoS One 7: e36345.

28. Carr LJ (2013) Multicomponent intervention to reduce daily sedentary time: a randomised controlled trial. BMJ open 3: e003261.

29. Columbia (2014) Inference for Means: Comparing Two Independent Samples 2014 [cited 2014 2015.120]; Available from: http://www.stat.ubc.ca/.

30. Joan Burton (2010) WHO Healthy Workplace Framework and Model : Background and Supporting Literature and Practices, Word Health Organization: Geneva, Switzerland

31. Schwarzer R, Schwarzer R (1992) Self-Efficacy in the Adoption and Maintenance of Health Behaviors: Theoretical Approaches and a New Model, in Self-Efficacy in the Adoption and Maintenance of Health Behaviors: Theoretical Approaches and a New Model, e.S.-E.T.C.o. Action, Editor. 1992, Hemisphere Publishing Corp: Washington DC: 214 .
32. Hall AG (2013) Incentives for healthy behaviors: experience from Florida Medicaid's Enhanced Benefit Rewards program. J Prim Care Community Health 4: 112-118.

33. Bann D (2015) Light Intensity Physical Activity and Sedentary Behavior in Relation to Body Mass Index and Grip Strength in Older Adults: Cross-Sectional Findings from the Lifestyle Interventions and Independence for Elders (LIFE) Study. PLOS ONE 10: e0116058.

34. Beddhu S (2015) Light-Intensity Physical Activities and Mortality in the United State General Population and CKD Subpopulation. Clinical Journal of the American Society of Nephrology 10: 1145-1153.

35. Haskell WL (2007) Physical activity and public health: updated recommendation for adults from the American College of Sports Medicine and the American Heart Association. Med Sci Sports Exerc 39: 1423-1434.

36. Healy GN (2008) Breaks in sedentary time: beneficial associations with metabolic risk Diabetes Care 31: 661-666.

37. Aittasalo M (2017) Moving to business - changes in physical activity and sedentary behavior after multilevel intervention in small and medium-size workplaces. BMC Public Health 17: 319

38. Mailey EL (2016) Comparing the effects of two different break strategies on occupational sedentary behaviour in a real-world setting: A randomized trial. Prev Med Rep 4: 423-428.

39. De Cocker K (2016) The Effectiveness of a Web-Based Computer-Tailored Intervention on Workplace Sitting: A Randomized Controlled Trial. J Med Internet Res 18: e96.

40. Kozey-Keadle S (2012) The Feasibility of Reducing and Measuring Sedentary Time among Overweight, Non-Exercising Office Workers. Journal of obesity 23: 282303.

41. Arrogi A (2017) Short- and long-term effectiveness of a three-month individualized need-supportive physical activity counseling intervention at the workplace. $B M C$ Public Health 17: 52.

42. Lu C (2008) Effects of an incentive-based online physical activity intervention on health care costs. Journal of occupational and environmental medicine/American College of Occupational and Environmental Medicine 50: 1209-1215.

43. Giles EL (2014) The effectiveness of financial incentives for health behaviour change: systematic review and meta-analysis. PloS one 9: e90347.

44. Paul-Ebhohimhen V, Avenell A (2008) Systematic review of the use of financial incentives in treatments for obesity and overweight. Obesity reviews: an official journal of the International Association for the Study of Obesity 9: 355-367.

45. Kinser PA, Robins JL (2013) Control Group Design: Enhancing Rigor in Research of Mind-Body Therapies for Depression. Evidence-Based Complementary and Alternative Medicine 12: 10.

46. Fukuoka Y (2011) The mPED randomized controlled clinical trial: applying mobile persuasive technologies to increase physical activity in sedentary women protocol. BMC public health 11: 933 .

47. Simon C (2014) A socio-ecological approach promoting physical activity and limiting sedentary behavior in adolescence showed weight benefits maintained 2.5 years after intervention cessation. International journal of obesity (2005) 38: 936-943.

48. Coffeng JK (2014) Effectiveness of a worksite social \& physical environmen intervention on need for recovery, physical activity and relaxation; results of a randomized controlled trial. PloS one 9: e114860.

49. Aadahl M (2014) Motivational counseling to reduce sitting time: a community-based randomized controlled trial in adults. American journal of preventive medicine 47: 576-586.

Copyright: (C2018 Zhou Z. This is an open-access article distributed under the terms of the Creative Commons Attribution License, which permits unrestricted use, distribution, and reproduction in any medium, provided the original author and source are credited. 